Editorial

\title{
Special Section on Cultural Heritage
}

Over the last two decades, there have been many high profile success stories where cutting edge computer graphics (CG) technology was used in collaboration with cultural heritage $(\mathrm{CH})$ professionals to unlock the secrets of humanity's legacy. Well known examples include the empirical 3D acquisition of Michelangelo's David, the laser scan-based 3D mapping of the tombs found in the Valley of the Kings and the decipherment of the instructions to and the operation of the 2nd-century BCE Antikythera Mechanismarguably the first computer in human kind history.

This special edition of Computers and Graphics contains new work that continues this tradition. Jenny and Hurni [1] propose a series of geometrical transformations that allow assessing the planimetric and geodetic accuracy of old maps before using the data for geo-historical studies. Laycock et al. [2] present techniques to aid in the semiautomatic extraction of building footprints from digital images of archive maps and sketches by aligning the old maps to modern vector data. Ducke et al. [3] propose an opensource pipeline for deriving a full 3D model from a series of overlapping images and illustrate it with data from the archaeological site at Weymouth. Osorio et al. [4] discuss novel digitising techniques for high specular reflection materials based on a multi-spectral approach and describe new virtual installations in the context of the Gold Museum in Bogota. Abel et al. [5] present a study of whether computed tomography is cost effective and can be used to capture and document the fine surface topology of flaked stone tools used by early humans. Li et al. [6] propose a skull completion framework based on symmetry and surface matching which will benefit subsequent archaeological and anthropological processing and analysis. Scheiblauer and Wimmer [7] present an out-of core interactive editing system for point clouds from a laser scanner. Finally, Blake and Ladeira [8] suggest a method for preparing users for a foreign cultural experience in Virtual Reality storytelling and illustrate it with San folklore.

More remarkable examples are sure to follow as leading $\mathrm{CH}$ institutions and professionals continue exploring advanced computer graphics-based tools and methods in collaboration with CG specialists. CG research on its own has pointed the way to new application development opportunities in the cultural heritage community. These collaborations and advanced CG research underscore the potential value of integrating robust digital imaging into ongoing $\mathrm{CH}$ working practice.

Regardless of these substantial potential advantages, little adoption of CG tools and methods within the $\mathrm{CH}$ community has taken place over the last twenty years. This slow rate of adoption is due to many factors. Many of the tools were and remain expensive and difficult to use by $\mathrm{CH}$ practitioners without extensive retraining and require the expertise of digital specialists from outside of the existing cultural heritage working cultures. The digital representations themselves often lacked transparency due to the absence of the digital equivalent of the traditional scientific 'lab notebook', documenting the means and circumstances of their generation. The hard truth is that most $\mathrm{CG}$ imaging of $\mathrm{CH}$ materials has focused exclusively on generating a high quality digital representation of the subject without providing a means for scientific evaluation of the representations quality. This led to a distrust of their scientific reliability and infrequent use of these CG representations in CH scholarship. Further, lack of process history transparency, dependence on closely held proprietary file formats and software, and an absence of established and funded long-term data preservation strategies, has led to concerns about digital data safety. Over the last twenty years, these concerns were confirmed by the increasing number of large-scale losses of digitally captured $\mathrm{CH}$ data, known today as the dawning of the Digital Dark Age. When paper records can last centuries, why adopt digital records that are useless after only ten years?

Only recently has the adoption rate of robust digital imaging tools by cultural heritage professionals begun to increase. This is partly due to CG driven work to remove the existing barriers to widespread adoption of digital technologies by adapting new robust imaging applications explicitly for use by cultural heritage professionals that are designed to be compatible with existing $\mathrm{CH}$ skill sets and working cultures. These applications, as well as related tool research and development road maps, include methods of enhancing scientific reliability of digital representations through the recording of scientific process history (digital 'lab notebooks'), and open source architectures that enhance long-term digital preservation strategies. Work in this volume examines the advantages of exclusive use of such open source workflows. Some of these computer graphics tools enable public access and interactive engagement both within collections and sites as well as over the Internet. Through the London and Seville Charters, the Virtual Reality (VR) community, made up of CG and CH professionals working together, has taken the lead in ensuring that transparency and the means for scientific qualitative evaluation are present in their work products. Museums have a growing interest in using VR representations to increase public access to their collections both within the museum and over the Internet. Several contributions to this Journal explore VR modalities to enrich the public experience of interaction with collections. These initiatives, along with the adoption by an increasing number of museums, libraries, archives, archaeologists, epigraphists, numismatists, and many others in the cultural heritage community of digital photography-based imaging tools and practices they can use by themselves, are creating an environment conducive to future widespread digital imaging adoption by $\mathrm{CH}$ professionals. 


\section{References}

[1] Bernhard Jenny, Lorenz Hurni. Studying cartographic heritage: analysis and visualization of geometric distortions. Computers and Graphics 2011;35(2):402-11. doi:10.1016/j.cag.2011.01.005.

[2] Stephen David Laycock, Philip G. Brown, Robert G. Laycock, Andy M. Day. Aligning archive maps and extracting footprints for analysis of historic urban environments. Computers and Graphics 2011;35(2):242-9. doi:10.1016/ j.cag.2011.01.002.

[3] Benjamin Ducke, David Score, Joseph Reeves. Multiview 3D reconstruction of the archaeological site at Weymouth from image series. Computers and Graphics 2011;35(2):375-82. doi:10.1016/j.cag.2011.01.006.

[4] Maria F. Osorio, Pablo Figueroa, Flavio Prieto, Pierre Boulanger, Eduardo Londoño. A novel approach at documenting artifacts at the Gold Museum in Bogota. Computers and Graphics 2011;35(4):894-903. doi:10.1016/j.cag.2011. 01.014 .

[5] Richard L. Abel, Simon A. Parfitt, Nick M. Ashton, Simon G. Lewis, Beccy Scott Chris B. Stringer. Digital preservation and dissemination of ancient lithic technology with modern micro-CT. Computers and Graphics 2011;35(4):878-84. doi:10.1016/j.cag.2011.03.001.

[6] Xin Li, Zhao Yin, Li Wei, Shenghua Wan, Wei Yu, Maoqing Li. Symmetry and template guided completion of damaged skulls. Computers and Graphics 2011;35(4):885-93. doi:10.1016/j.cag.2011.01.015.

[7] Claus Scheiblauer, Michael Wimmer. Out-of-core selection and editing of huge point clouds. Computers and Graphics 2011;35(2):342-51. doi:10.1016/j.cag. 2011.01.004.

[8] Edwin Blake, Ilda Ladeira. Cultural reinterpretation and resonance: the san and hip-hop. Computers and Graphics 2011;35(2):383-91. doi:10.1016/ j.cag.2011.01.003.

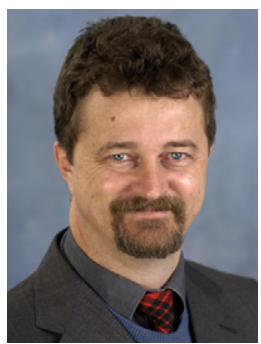

Alan Chalmers is Professor of Visualisation in the International Digital Laboratory, WMG, at the University of Warwick. He has published over 190 papers in journals and international conferences on realistic computer graphics, parallel processing, multi-sensory perception and virtual archaeology. He is Honorary President of Afrigraph and a former Vice President of ACM SIGGRAPH. In addition, he is Founder and Innovation Director of the spinout company, goHDR, which is developing software to facilitate the widespread adoption of high dynamic range (HDR) imaging technology. His research is working towards achieving Real Virtuality: high-fidelity, multi-sensory virtual environments, including developing "digital taste".

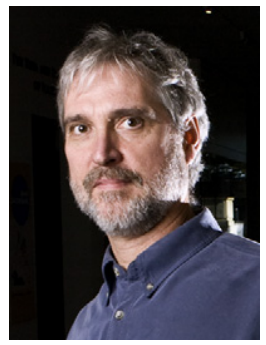

Mark Mudge is President and co-founder of Cultura Heritage Imaging ( $\mathrm{CHI}$ ), a public charity and California non-profit corporation, incorporated in 2002. Mark has BA in Philosophy from New College of Florida (1979). He has worked as a professional bronze sculptor and has been involved in photography and 3D imaging for over 20 years. He is a co-inventor, with Tom Malzbender, of the computational photography technique, Highlight Reflectance Transformation Imaging. He has published twelve articles and book chapters related to imaging cultural heritage materials and serves on several international committees, including The International Council of Museums' (ICOM) Documentation Committee (CIDOC).

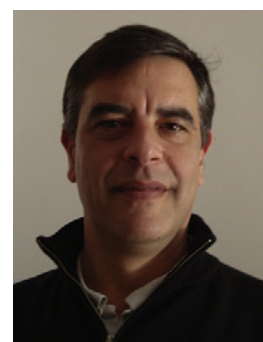

Luis Paulo Santos is an Auxiliar Professor at the Department of Informatics, Universidade do Minho, Portugal. His research interests are in interactive high fidelity rendering and parallel processing. He received his Ph.D. in 2001 from Universidade do Minho in Scheduling under Conditions of Uncertainty. Luis Paulo published several papers on both computer graphics and parallel processing on international conferences and journals. He has been a member of several international program committees, acted as program cochair of the 2007 EGPGV symposium and EuroPar 2005 conference and organized EGPGV 2006, VAST 2008 and VS-Games 2010 in Braga, Portugal. He manages several nationally funded graphics R\&D projects and participates in several European projects with both academia and industry. He is a member of the Direction Board of the Portuguese chapter of Eurographics since 2008.

Guest Editors

Alan Chalmers

Visualisation in the International Digital Laboratory, WMG, University of Warwick, UK E-mail address: Alan.Chalmers@warwick.ac.uk

Mark Mudge Cultural Heritage Imaging (CHI), California, USA

Luis Paulo Santos*

Department of Informatics, Universidade do Minho, Portugal E-mail address: psantos@di.uminho.pt

Received 31 March 2011 